\begin{tabular}{|c|l|}
\hline Title & Electron dynamics in chromium probed with 20-fs optical pulses \\
\hline Author(s) & Hirori, H.; Tachizaki, T.; Matsuda, O.; Wright, O. B. \\
\hline Citation & $\begin{array}{l}\text { PHY SICAL REVIEW B, 68, 113102 } \\
\text { https://doi.org/L0.1103/PhysRevB.68.113102 }\end{array}$ \\
\hline Issue Date & 2003-09-16 \\
\hline Doc URL & http://hdl.handle.net/2115/5788 \\
\hline Rights & Copyright $\odot 2003$ A merican Physical Society \\
\hline Type & article \\
\hline File Information & PRB68.pdf \\
\hline
\end{tabular}

Instructions for use 


\title{
Electron dynamics in chromium probed with 20 -fs optical pulses
}

\author{
H. Hirori, T. Tachizaki, O. Matsuda, and O. B. Wright* \\ Department of Applied Physics, Faculty of Engineering, Hokkaido University, Sapporo 060-8628, Japan
}

(Received 17 March 2003; published 16 September 2003)

\begin{abstract}
Electron excitation and relaxation in chromium are probed with 20-fs time resolution using an ultrafast optical technique. We obtain good fits to the data for the transient reflectivity and transmittivity changes in a thin film using a simple model of electron relaxation, suggesting the existence of an efficient electron-electron thermalization process on ultrashort-time scales. Quantitative analysis allows the extraction of thermo-optic coefficients and dielectric constant variations related to both the electron and the lattice temperatures.
\end{abstract}

DOI: 10.1103/PhysRevB.68.113102

PACS number(s): 78.47.+p, 78.20.Nv, 78.20.Ci

Nonequilibrium electron distributions can be excited in metals with an ultrashort light pulse. ${ }^{1}$ The subsequent energy exchange between the electrons and the lattice is governed predominantly by the electron-phonon (e-p) scattering time, but is also affected by the electron-electron (e-e) scattering time. The noble metals, possessing simple band structures, have provided a fertile testing ground for theories of nonequilibrium electron relaxation and diffusion. ${ }^{2-8}$ It is now possible to investigate the electron dynamics on time scales of the order of the e-e scattering time, typically $10-50 \mathrm{fs}$ for excess electron energies $\sim 1 \mathrm{eV}$. Although in the noble metals and in the alloy $\mathrm{CoPt}_{3}$, where the e-p interaction is relatively weak, the evolution of such transient athermal electron distributions was investigated with $\sim 20$-fs time resolution, ${ }^{7-9}$ in other transition metals no studies have been made on these time scales, to the best of our knowledge.

The group-VIB transition metals $(\mathrm{Cr}, \mathrm{Mo}, \mathrm{W})$ are interesting because they have large values of the e-p coupling constant, resulting in short electron energy relaxation times $\sim 200$ fs compared to the noble metals $(\sim 1 \mathrm{ps}) .{ }^{10}$ Their band structure is complicated, there being a significant density of states due to $d$ electrons in the region around the Fermi level. ${ }^{11}$ Understanding the short-time electron dynamics in such metals with strong e-p coupling should become essential for applications in future ultrafast devices with ultrahigh switching speeds. The electron relaxation in thin films of chromium and tungsten under spatially homogeneous conditions was measured by Brorson et al. with optical pulses of duration $60 \mathrm{fs} .{ }^{10}$ But only the reflectivity change was probed, thus preventing access to the transient dielectric constant. In this report we monitor both the reflectivity and transmittivity changes in a thin polycrystalline film of chromium with 20 -fs optical pulses to elucidate the ultrafast dynamics of the electrons therein.

The film of $\mathrm{Cr}$ on a crown glass substrate is excited and probed from the front side with near Fourier-transformlimited optical pulses from a Ti:sapphire laser (KapteynMurmane Labs) of central wavelength $790 \mathrm{~nm}$, repetition rate $87 \mathrm{MHz}$, pulse duration $\tau_{L}=20 \mathrm{fs}$ [full width at half maximum (FWHM) intensity], and spectral width $\sim 50 \mathrm{~nm}$ (FWHM). A schematic diagram of the apparatus is shown in Fig. 1. Interband transitions of electrons within the photon energy $(1.57 \mathrm{eV})$ of the Fermi surface $\left(\right.$ at $\left.\varepsilon_{F} \approx 7 \mathrm{eV}\right)$ are primarily induced. ${ }^{11}$ The optical pulse duration is controlled by two prisms. It is measured at the sample position by a noncollinear cross-correlation technique, through the temporary insertion of a $25-\mu \mathrm{m}$ slab of beta barium borate that combines the cross-polarized pump and probe beams at $0^{\circ}$ and $10^{\circ}$ ( $p$-polarized) incidence, respectively. At the sample the optical spot radius at $1 / e$ intensity is $a \approx 9.5 \mu \mathrm{m}$, the typical incident pump pulse energy is $E=0.75 \mathrm{~nJ}$ (maximum incident fluence $E / \pi a^{2} \approx 0.26 \mathrm{~mJ} \mathrm{~cm}^{-2}$ ), and the probe pulse energy is $0.007 \mathrm{~nJ}$. The predicted electron temperature change (for a thermalized electron gas) is $220 \mathrm{~K}$ and that of the lattice is $8 \mathrm{~K}$. The pump light is chopped mechanically at $2 \mathrm{kHz}$, and a synchronous signal proportional to the reflected or transmitted intensity variation of the probe light is fed to a lock-in amplifier. Multiple scanning with an optical delay line allows a resolution for relative intensity changes $\sim 5$ $\times 10^{-6}$ (with a total integration time of $\sim 2 \mathrm{~s}$ per point with 280 points, using 60 scans).

The polycrystalline Cr sample of thickness $d_{0}=19 \mathrm{~nm}$ is prepared on a crown glass substrate of thickness $1 \mathrm{~mm}$ at a pressure of $5 \times 10^{-6}$ Torr by electron-beam deposition at 0.3 $\mathrm{nm} \mathrm{s}^{-1} .{ }^{12}$ The grain size was estimated by atomic force microscopy to be $100-200 \mathrm{~nm}$. The thickness is measured by spectroscopic ellipsometry and confirmed with picosecond ultrasonics. ${ }^{13}$ The refractive index of the film $(n+i \kappa=3.65$

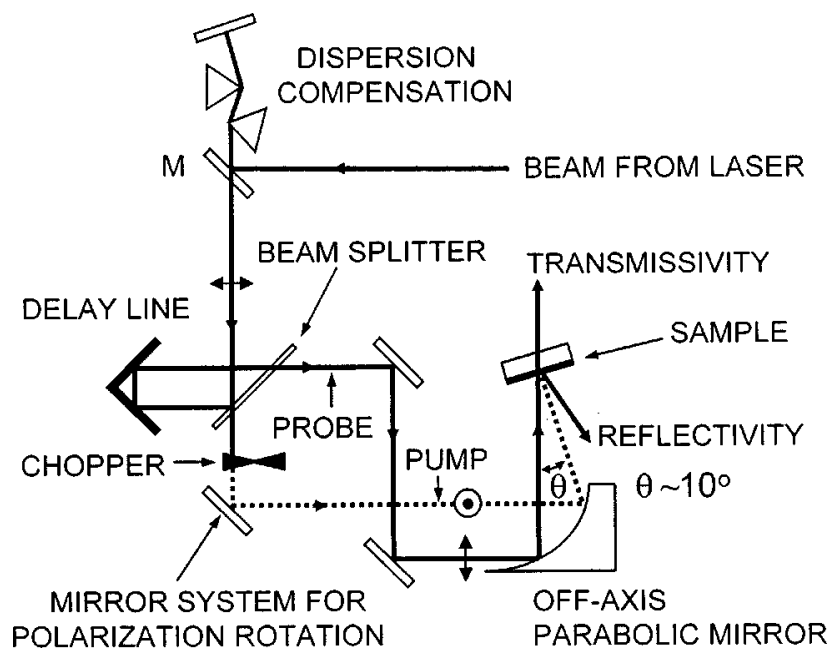

FIG. 1. Schematic diagram of the experimental setup. The beam exiting from the dispersion-compensation prisms passes below the mirror M. The first reflection from the beam splitter, a glass slide of thickness $1 \mathrm{~mm}$, is used for the probe. 


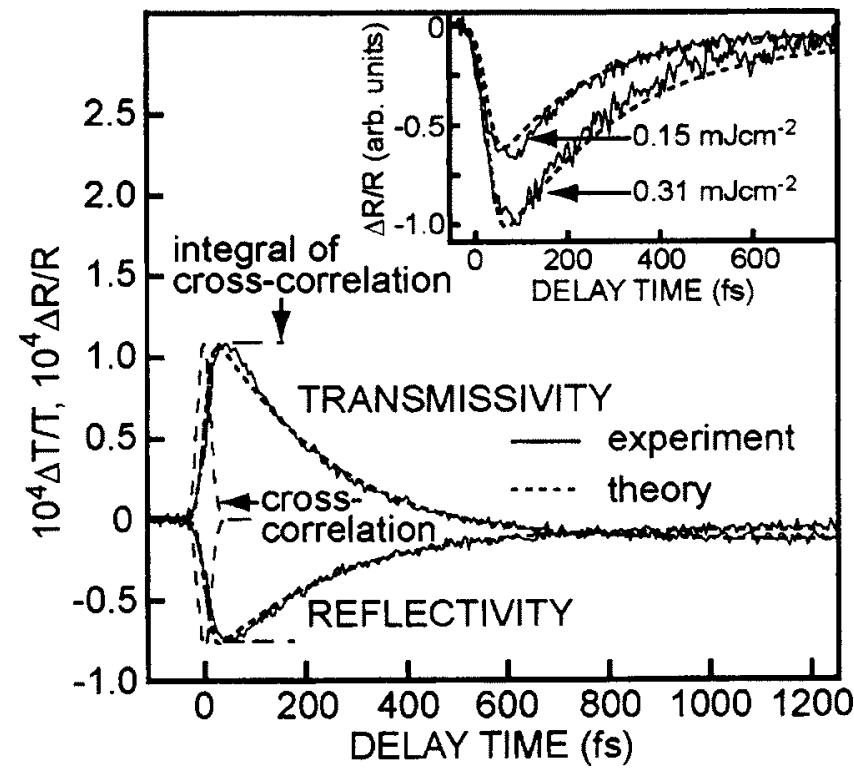

FIG. 2. Comparison of the relative reflectivity and transmissivity changes, $\Delta R / R$ and $\Delta T / T$, measured (solid curves) for a 19-nm Cr film with FWHM optical pulse duration $\tau_{L}=20 \mathrm{fs}$ at $1.57 \mathrm{eV}$ $(790 \mathrm{~nm})$, and calculated (dotted curves) using the two-temperature model. The pump fluence is $0.26 \mathrm{~mJ} \mathrm{~cm}^{-2}$. Also shown are the pump-probe cross correlation (dashed curve) and its integral (dashdotted curve). The inset shows a comparison of the relative reflectivity changes measured (solid curves) and fitted (dotted curvesfitted simultaneously with the same values for $a, b, c, d$, and $g$ as above and appropriate lattice temperatures) for $\tau_{L}=34 \mathrm{fs}$ using the two-temperature model for pump fluences 0.31 and $0.15 \mathrm{~mJ} \mathrm{~cm}^{-2}$, respectively.

$+3.09 i)$ and substrate $\left(n_{s}=1.52\right)$ at $790 \mathrm{~nm}$ are also measured by ellipsometry. This value of $\kappa$ corresponds to a 20-nm optical-absorption depth. The measured static reflection and transmission coefficients of the sample for the ultrashort optical pulses are, respectively, $R \approx 0.46$ and $T$ $\approx 0.16$. These values are close to those $(R \approx 0.43$ and $T$ $\approx 0.12$ ) expected from the measured $n+i \kappa$ for normally incident monochromatic light at $790 \mathrm{~nm}$. We expect a nearly homogeneous (to within 10\%) optical-absorption profile in the depth direction in the film. With the steady-state sample temperature in the probed region estimated at $\approx 470 \mathrm{~K},{ }^{14} \mathrm{Cr}$ is in its paramagnetic state. (Exposure to a temperature of this order during the experiment is not expected to significantly increase the native oxide layer thickness on the sample, of the order of a few $\mathrm{nm}$ in thickness, that should have a negligible effect on the present measurements. ${ }^{15}$ )

Figure 2(a) shows the experimental relative reflectivity and transmittivity changes (solid curves) as a function of delay time. The initial change in the signals is compared with the integral (dash-dotted curves) of the cross correlation (dashed curves) for the pump and probe optical pulses. The data follow closely behind this integral in the initial stages. The sign of $\Delta R$ (dependent on film thickness ${ }^{16}$ ) is opposite to that of $\Delta T$. The simplest theoretical model that can account for this data reasonably well is the two-temperature model: ${ }^{17}$ the electrons and the lattice are assumed to be described by separate temperatures $T_{e}$ and $T_{l}$, coupled by the e-p interaction. In a one-dimensional approximation, valid since $a \gg d_{0}$,

$$
\begin{gathered}
C_{e}\left(T_{e}\right) \frac{\partial T_{e}}{\partial t}=-g\left(T_{e}-T_{l}\right)+S(z, t), \\
C_{l} \frac{\partial T_{l}}{\partial t}=g\left(T_{e}-T_{l}\right),
\end{gathered}
$$

where $z$ is the depth coordinate. The e-p coupling constant $g$ and the lattice heat capacity $C_{l}$ are assumed to be constant because of the relatively small transient change in lattice temperature $(\sim 10 \mathrm{~K}))^{1,18}$ The electron heat capacity, about 35 times smaller than $C_{l}\left(\approx 3.6 \mathrm{Jm}^{-3} \mathrm{~K}^{-1}\right.$ at $\left.470 \mathrm{~K}\right)$, is given by $C_{e}\left(T_{e}\right)=\gamma T_{e}\left(\gamma=193 \mathrm{Jm}^{-3} \mathrm{~K}^{-2}\right) .{ }^{19}$ The source term due to the optical pulse is taken as

$$
S(t)=2 K \sqrt{\frac{\ln 2}{\pi}} \frac{E}{\pi a^{2}} \frac{(1-R-T)}{d_{0} \tau_{L}} \exp \left[-4 \ln 2\left(\frac{t}{\tau_{L}}\right)^{2}\right],
$$

where the factor $K=\frac{1}{2}$ here is calculated to account for the spot size of the probe beam. Because of the poor air and substrate thermal diffusivities, we assume no transport of heat (or electrons) across the film boundaries on our experimental time scales.

Neglecting the small modulation from the substrate on our time scales, the reflectance $(r)$ and transmittance $(\tau)$ changes $(\ll 1)$ for the present geometry can be calculated from the transient dielectric constant change $\Delta \varepsilon=\Delta(n+i \kappa)^{2}$ of the film by assuming monochromatic incident radiation and spatially homogeneous modulation: ${ }^{20}$

$$
\begin{gathered}
\frac{\delta r}{r}=\frac{i k_{0} \Delta \varepsilon}{2 a_{0} b_{0}} \int_{0}^{d_{0}}\left(a_{1} e^{i k_{1} z}+b_{1} e^{-i k_{1} z}\right)^{2} d z \\
\frac{\delta \tau}{\tau}=\frac{i k_{0} \Delta \varepsilon}{2 a_{0}} \int_{0}^{d_{0}}\left(a_{1} e^{i k_{1} z}+b_{1} e^{-i k_{1} z}\right)\left(a_{1}^{\prime} e^{i k_{1} z}+b_{1}^{\prime} e^{-i k_{1} z}\right) d z,
\end{gathered}
$$

where $k_{0}$ and $k_{1}$ are wave numbers and $a_{0}, b_{0}, a_{1}, b_{1}, a_{1}^{\prime}$, and $b_{1}^{\prime}$ are constants, ${ }^{21}$ for which 0 refers to air and 1 to the film. The measured relative reflectivity $(\Delta R / R)$ and transmittivity $(\Delta T / T)$ changes are related to Eqs. (3) and (4) by $\Delta R / R=2 \operatorname{Re}(\delta r / r), \Delta T / T=2 \operatorname{Re}(\delta \tau / \tau)$ [since $R=|r|^{2}$ and $T$ $\left.=|t|^{2}\right]$. Here, $\Delta \varepsilon$ is assumed to be linearly related to the electron and lattice temperature changes for our range of fluences. Measurements and fits for $\Delta R$ at different pump fluences (with $\tau_{L}=34 \mathrm{fs}$-see Fig. 2 inset) confirm that this first-order approximation is reasonable: ${ }^{22}$

$$
\Delta \varepsilon=\Delta \varepsilon_{1}+i \Delta \varepsilon_{2}=\left(a \Delta T_{e}+b \Delta T_{l}\right)+i\left(c \Delta T_{e}+d \Delta T_{l}\right),
$$

where $a=\partial \varepsilon_{1} / \partial T_{e}, \quad b=\partial \varepsilon_{1} / \partial T_{l}, \quad c=\partial \varepsilon_{2} / \partial T_{e}, \quad$ and $d$ $=\partial \varepsilon_{2} / \partial T_{l}$. We treat these thermo-optic coefficients and $g$ as fitting parameters, substituting the numerical solution of the nonlinear Eqs. (1) and (2) obtained by the finite-difference technique into Eqs. (3)-(5). ${ }^{23}$ By least-squares fitting using parameters $a, b, c, d$, and $g$ common to the two data curves in 


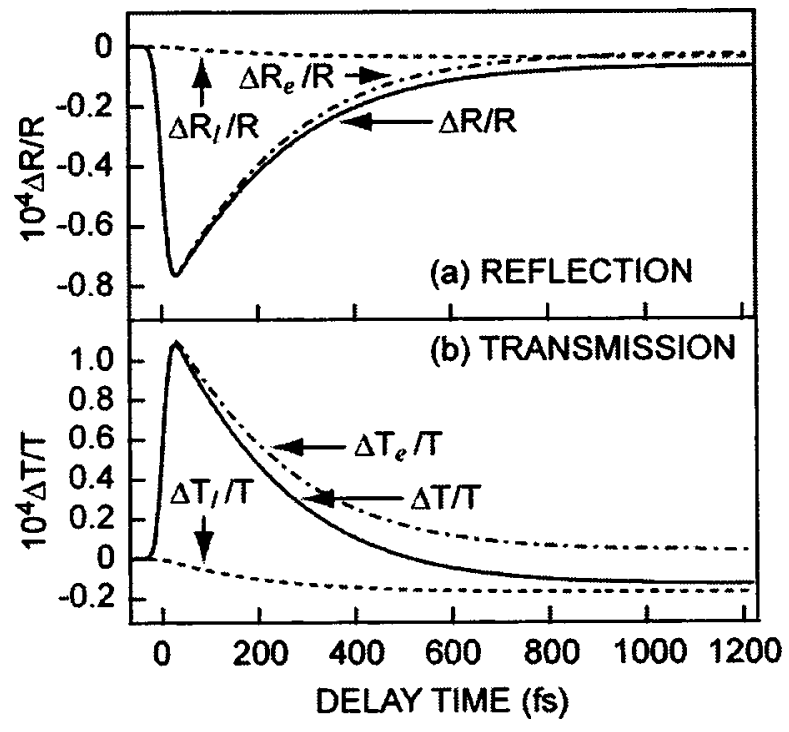

FIG. 3. (a) Calculated evolution of the relative reflectivity change $\Delta R / R$ (solid curve), and the separate electronic and lattice contributions to $\Delta R / R: \Delta R_{e} / R$ (dash-dotted curve) and $\Delta R_{l} / R$ (dashed curve), respectively. (b) Calculated evolution of the relative transmissivity $\Delta T / T$ (solid curve), and the electronic and lattice contributions to $\Delta T / T: \Delta T_{e} / T$ (dash-dotted curve) and $\Delta T_{l} / T$ (dashed curve), respectively. (In this figure only, $\Delta T_{e}$ and $\Delta T_{l}$ refer to changes in transmissivity rather than in temperature.)

Fig. 2, and including a convolution with the probe pulse temporal profile, theoretical curves (dotted lines) are obtained that are very similar to experiment. This relative success of the two-temperature model may well extend to a wide range of optical wavelengths, considering the broad features in the joint density of states of the electrons in the VIB transition metals. ${ }^{11,24}$

The fitted value of $g=(470 \pm 30) \times 10^{15} \mathrm{Jm}^{-3} \mathrm{~K}^{-1}$ is in good agreement with (relatively temperature-insensitive ${ }^{6}$ ) literature values. ${ }^{10,25}$ The thermo-optic coefficients obtained are $\quad \partial \varepsilon_{1} / \partial T_{e}=-2.4 \times 10^{-5}, \quad \partial \varepsilon_{1} / \partial T_{l}=-41 \times 10^{-5}$, $\partial \varepsilon_{2} / \partial T_{e}=-1.2 \times 10^{-5}$, and $\partial \varepsilon_{2} / \partial T_{l}=2.0 \times 10^{-5}$ in units of $\mathrm{K}^{-1}$. (The uncertainties are typically $\pm 30 \%$, owing mainly to the errors in the measurement of the pump fluence and in the multiparameter fitting.) These coefficients, sensitive to the band structure, are strong functions of wavelength. ${ }^{11,26}$ Our low value for the ratio $\left(\partial \varepsilon_{2} / \partial T_{l}\right.$ $\left.+\partial \varepsilon_{2} / \partial T_{e}\right) /\left(\partial \varepsilon_{1} / \partial T_{l}+\partial \varepsilon_{1} / \partial T_{e}\right)$ at $790 \mathrm{~nm}$ is consistent with that found in low-frequency thermomodulation measurements, ${ }^{26}$ although the comparison is not exact because our experiments are done under conditions of approximately constant (zero) strain as opposed to constant (zero) stress.

The experimental $\Delta R / R$ and $\Delta T / T$ variations are difficult to interpret physically in terms of microscopic theories. The transient dielectric constant variation is more fundamental and is directly related to changes in the density of states governing the relevant electronic transitions. For our homogeneously excited thin film, the changes in $\Delta R$ and $\Delta T$ are linearly related to those in the dielectric constants: ${ }^{4,5,8}$

$$
\Delta R / R=\alpha_{1} \Delta \varepsilon_{1}+\alpha_{2} \Delta \varepsilon_{2},
$$

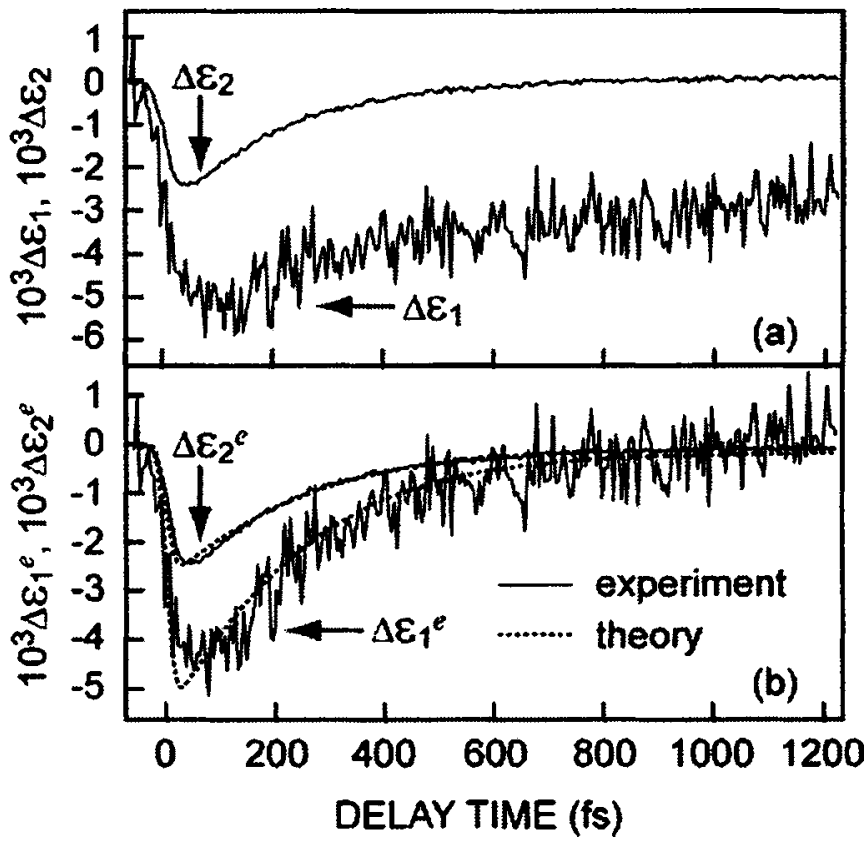

FIG. 4. (a) Measured transient changes of the real and imaginary parts $\Delta \varepsilon_{1}$ and $\Delta \varepsilon_{2}$ of the dielectric constant. (b) The extracted experimental electronic contributions to $\Delta \varepsilon: \Delta \varepsilon_{1}^{e}$ and $\Delta \varepsilon_{2}^{e}$ (solid curves). Theoretical curves (dotted) are also shown.

$$
\Delta T / T=\beta_{1} \Delta \varepsilon_{1}+\beta_{2} \Delta \varepsilon_{2},
$$

where the coefficients $\alpha_{1}, \alpha_{2}, \beta_{1}$, and $\beta_{2}$ can be obtained from Eqs. (3) and (4). Knowing the fitted $\Delta R / R$ and $\Delta T / T$ variations allows one to solve for the theoretical $\Delta \varepsilon_{1}$ and $\Delta \varepsilon_{2}$ variations, and in turn from Eqs. (3)-(5) for the theoretical electronic and lattice contributions to $\Delta R / R$ and $\Delta T / T$, as shown in Fig. 3 (based on values of $\alpha_{1}, \alpha_{2}, \beta_{1}$ and $\beta_{2}$ equal to $0.0025,0.024,0.0037$ and -0.050 , respectrively). The lattice contribution for both the relative reflectivity [Fig. 3(a)] and transmissivity [Fig. 3(b)] changes tends to a constant value as the electron temperature relaxes. The contribution from the electron temperature change is dominant at short times in both cases.

Equations (6) and (7) also allow one to derive the experimental $\Delta \varepsilon_{1}$ and $\Delta \varepsilon_{2}$ variations (independent of any dynamical model), as shown in Fig. 4(a). Some salient points regarding these are as follows: (i) the temporal shape of $\Delta \varepsilon_{2}$ is similar to that of $\Delta R / R$ and $\Delta T / T$, and $\Delta \varepsilon_{2}$ is dominated by the electronic contribution; (ii) the temporal shape of $\Delta \varepsilon_{1}$ is different, and is strongly influenced by the lattice contribution; (iii) the noise level for $\Delta \varepsilon_{1}$ is significantly larger than that for $\Delta \varepsilon_{2}$. The results are a direct consequence of the signs and relative magnitudes of $\alpha_{1}, \alpha_{2}, \beta_{1}, \beta_{2}$ and of $a, b, c, d$. (iv) The magnitude of $\Delta \varepsilon_{1}$ is larger than that of $\Delta \varepsilon_{2}$, both quantities being negative. The sign of $\Delta \varepsilon_{2}$ indicates a decrease in absorption during the transient heating. The derived electronic contributions to the experimental dielectric constant variations, $\Delta \varepsilon_{1}^{e}$ and $\Delta \varepsilon_{2}^{e}$, are shown in Fig. 4(b). Comparison with the predictions of the twotemperature model (dashed curves) shows good agreement at times $t>100 \mathrm{fs}$, suggesting that $T_{e}$ is well defined at these 
times and that the electron distribution is internally thermalized within $\sim 100$ fs by e-e scattering. By fitting the experimental $\Delta \varepsilon^{e}$ decays to the approximate function exp $\left(-t / \tau_{1,2}^{e}\right)$, the effective electron energy relaxation times $\tau_{1}^{e}$ for $\Delta \varepsilon_{1}^{e}(t)$ and $\tau_{2}^{e}$ for $\Delta \varepsilon_{2}^{e}(t)$ are found to be $230 \pm 50$ and $210 \pm 20 \mathrm{fs}$, respectively. These values are longer than the value $\tau_{e}\left(T_{e}=T_{l}=470 \mathrm{~K}\right)=C_{e} / g \approx 190 \mathrm{fs}$ expected for the same initial lattice temperature in the limit of small $\Delta T_{e}$. This increase in $\tau_{e}$ with increasing $T_{e}$ is in agreement with rough estimates based on an approximate first-order solution to Eqs. (1) and (2) at constant $T_{l}: \tau_{e} \approx \tau_{e}\left(T_{l}\right)\left(1+T_{e} / T_{l}\right) / 2$ (see Ref. 2). In a more sophisticated treatment the effect of the finite e-e relaxation time ${ }^{27}$ and the band structure ${ }^{11}$ should be taken into account. The variations $\Delta \varepsilon_{1}^{e}(t)$ and $\Delta \varepsilon_{2}^{e}(t)$ arise from a sum of all allowed electronic transitions at the probe wavelength originating in different regions of $\mathbf{k}$ space whose populations are perturbed by the pump optical pulse. These variations cannot be ascribed to a single interband transition in the complex band structure of $\mathrm{Cr}^{11,26} \mathrm{~A}$ more detailed analysis of the $\Delta \varepsilon$ variations is beyond the scope of this report.

In conclusion we have investigated the nonequilibrium dynamics of electron relaxation in chromium with 20 -fs time resolution using an ultrafast optical technique. The twotemperature model produces good agreement with the experimental data, implying electron-electron thermalization within $\sim 100$ fs in this metal. This efficient thermalization and the short electron energy relaxation time in chromium bode well for future applications in ultrafast control of charge distributions and transport in transition metals.

We are grateful to Vitali Gusev and Hrvoje Petek for valuable discussions.
*Corresponding author. Electronic address: assp@kinoap.eng.hokudai.ac.jp

${ }^{1}$ G. L. Eesley, Phys. Rev. B 33, 2144 (1986).

${ }^{2}$ V. E. Gusev and O. B. Wright, Phys. Rev. B 57, 2878 (1998).

${ }^{3}$ S. D. Brorson, J. G. Fujimoto, and E. P. Ippen, Phys. Rev. Lett. 26, 1962 (1987).

${ }^{4}$ C. K. Sun, F. Vallee, L. H. Acioli, E. P. Ippen, and J. G. Fujimoto, Phys. Rev. B 50, 15337 (1994).

${ }^{5}$ S. Ogawa, H. Nagano, and H. Petek, Phys. Rev. B 55, 10869 (1997).

${ }^{6}$ R. H. M. Groeneveld and R. Sprik, Phys. Rev. B 51, 11433 (1995).

${ }^{7}$ N. Del Fatti, R. Bouffanais, F. Vallee, and C. Flytzanis, Phys. Rev. Lett. 81, 922 (1998).

${ }^{8}$ N. Del Fatti, C. Voisin, M. Acherman, S. Tzortzakis, D. Christofilos, and F. Vallee, Phys. Rev. B 61, 16956 (2000).

${ }^{9}$ L. Guidoni, E. Beaurepaire, and J. Bigot, Phys. Rev. Lett. 89, 017401 (2002).

${ }^{10}$ S. D. Brorson, A. Kazeroonian, J. S. Moodera, D. W. Face, T. K. Cheng, E. P. Ippen, M. S. Dresselhaus, and G. Dresselhaus, Phys. Rev. Lett. 18, 2172 (1990).

${ }^{11}$ E. Colavita, A. Franciosi, C. Mariani, and R. Rosei, Phys. Rev. B 27, 4684 (1983).

${ }^{12}$ Another sample of similar thickness made by the same technique at $5 \times 10^{-7}$ Torr and $0.7 \mathrm{~nm} \mathrm{~s}^{-1}$ gave an identical reflectivity response, allowing us to conclude that the residual oxygen content of our film did not influence the results. See A. K. Kulkarni and L. C. Chang, Thin Solid Films 301, 17 (1997).

${ }^{13}$ D. H. Hurley and O. B. Wright, Opt. Lett. 24, 1305 (1999).

${ }^{14}$ This value, averaged over the probe beam profile, is estimated from a three-dimensional heat conduction model using the average absorbed pump power $(12.4 \mathrm{~mW})$, the Gaussian pump spot radius $a$, and the thermal conductivities of the substrate (1.4 $\left.\mathrm{Wm}^{-1} \mathrm{~K}^{-1}\right)$ and film $\left(94 \mathrm{Wm}^{-1} \mathrm{~K}^{-1}\right)$.

${ }^{15}$ E. Idczak and E. Oleszkiewicz, Opt. Spetkrosk. 49, 570 (1980) [Opt. Spectrosc. 49, 309 (1980)].

${ }^{16}$ J. L. Hosteler, A. N. Smith, D. M. Czajkowsky, and P. M. Norris, Appl. Opt. 38, 3614 (1999).

${ }^{17}$ S. I. Anisimov, B. L. Kapeliovich, and T. L. Perel'man, Zh. Eksp. Theor. Phys 66, 776 (1974) [Sov. Phys. JETP 39, 375 (1974)].

${ }^{18}$ T. Q. Qui and C. L. Tien, J. Heat Transfer 115, 835 (1993).

${ }^{19}$ C. Kittel, Introduction to Solid State Physics, 7th ed. (Wiley, New York, 1996).

${ }^{20}$ O. Matsuda and O. B. Wright, J. Opt. Soc. Am. B 19, 3028 (2002).

${ }^{21}$ Here $k_{0}, k_{1}$, and $k_{2}$ are the wave numbers in the air, film, and substrate, respectively, and $a_{0}=\left(k_{0}+k_{1}\right)\left(k_{1}+k_{2}\right)$ $+\left(k_{0}-k_{1}\right)\left(k_{1}-k_{2}\right) e^{2 i k_{1} d_{0}}, \quad b_{0}=\left(k_{0}-k_{1}\right)\left(k_{1}+k_{2}\right)+\left(k_{0}+k_{1}\right)$ $\times\left(k_{1}-k_{2}\right) e^{2 i k_{1} d_{0}}, \quad a_{1}=2 k_{0}\left(k_{1}+k_{2}\right), \quad b_{1}=2 k_{0}\left(k_{1}-k_{0}\right) e^{2 i k_{1} d_{0}}$, $a_{1}^{\prime}=\left(k_{1}-k_{0}\right) / 2 k_{1}$, and $b_{1}^{\prime}=\left(k_{0}+k_{1}\right) / 2 k_{1}$.

${ }^{22}$ The measurements in the inset of Fig. 2 were also repeated in the range $0-150 \mathrm{fs}$ at higher resolution to check the short-time response at different fluences. The characteristic slightly delayed response at short times compared to the two-temperature model was more clearly evident at both fluences.

${ }^{23}$ A. N. Smith, J. L. Hostetler, and P. M. Norris, Numer. Heat Transfer, Part A 35, 859 (1999).

${ }^{24}$ W. B. Pickett and P. B. Allen, Phys. Rev. B 11, 3599 (1975).

${ }^{25}$ T. Q. Qiu and C. L. Tien, Int. J. Heat Mass Transfer 37, 2789 (1994).

${ }^{26}$ E. Colavita, A. Franciosi, D. W. Lynch, G. Paolucci, and R. Rosei, Phys. Rev. B 27, 1653 (1983).

${ }^{27}$ V. P. Zhukov, F. Aryasetiawan, E. V. Chulkov, and P. M. Echenique, Phys. Rev. B 65, 115116 (2002). 